\section{Quicker reaction by design}

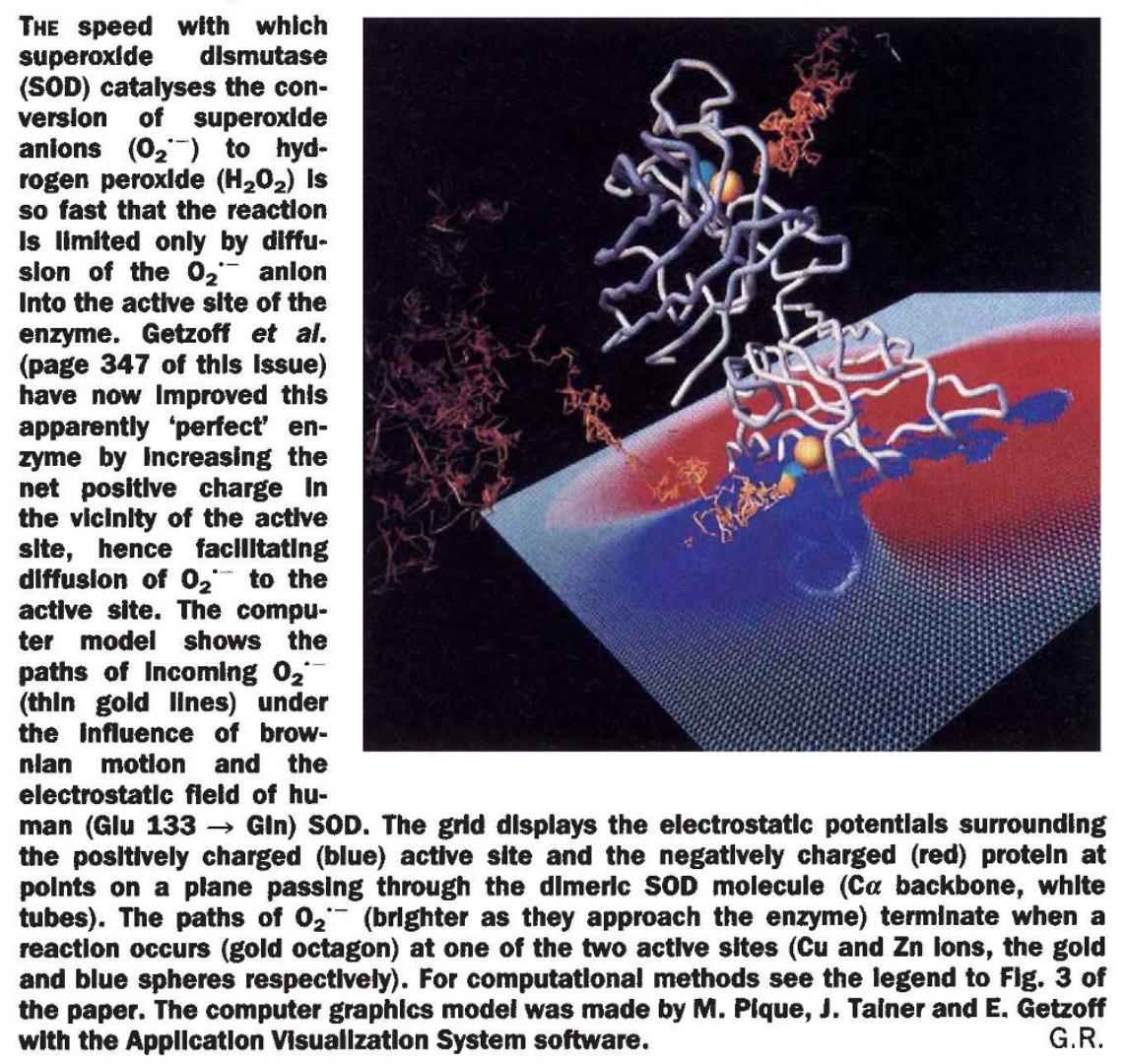

frequency of the photolysis laser, and hence the photon energy supplied to ketene molecules, is varied. The PHOFEX signals showed steps matching those in the $k(E)$ curves, consistent with the expectation that it is changes in $k(E)$ which largely determine the structure in the PHOFEX spectra.

What are the crucial features alluded to earlier that allow Moore and colleagues' experiment to uncover the structure in the transition state associated with ketene dissociation? The first, which is crucial in many modern experiments in spectroscopy and reaction dynamics, is the cooling of the reagents provided by the jet expansion. Strictly, the microcanonical rate constant depends not only on the internal energy of the reagent but also on its state of total angular momentum, $J$, as this must be retained during unimolecular fragmentation. Jet cooling severely restricts the $J$ states populated by ketene molecules, with the result that the averaging over different $k(E, J)$ curves is minimal and it does not obscure the structure in $k(E)$ resulting from the pattern of quantum levels in the transition state.

The second, and perhaps even more important aspect of the study is related to the special features of the ketene dissociation. As Fig. 1 indicates, there is a well-defined energy barrier along the path leading to the dissociation products.
This is not always the case for a unimolecular reaction, and it leads to a transition state which is fairly tightly constrained and which consequently has rather well-separated quantum levels. The lowest frequency modes of motion, which lead to the closest-spaced quantum levels and hence to the most closely spaced steps in $k(E)$, are the bending of the $\mathrm{C}-\mathrm{C}-\mathrm{O}$ angle and the torsional motions of the $\mathrm{CH}_{2}$ and $\mathrm{CO}$ moieties around the breaking $\mathrm{C}-\mathrm{C}$ bond. The torsional motion becomes an internal rotation at energies of about $400 \mathrm{~cm}^{-1}$. As a result, energy levels associated with this motion become much more closely packed and this may be the reason for the rapid, and essentially unstructured, increase in $k(E)$ at the higher values of $E$ which were examined.

Another interesting characteristic of the results is that, in principle, the rate constant for dissociation at threshold should correspond to that associated with passage through the single, lowest level in the transition state, so that $k\left(E^{0}\right)$ $=1 / h \rho\left(E^{0}\right)$. Consequently, it should be possible to compare the density of reagent states at threshold derived from the experimental value of $k\left(E^{0}\right)$ and the value estimated from a knowledge of the frequencies and anharmonicities of the vibrations of $\mathrm{CH}_{2} \mathrm{CO}$. In practice, there is some uncertainty about the degeneracy of the transition-state quantum levels. But Moore and coworkers performed experiments on $\mathrm{CD}_{2} \mathrm{CO}$, as well as on $\mathrm{CH}_{2} \mathrm{CO}$, and find a fourfold reduction in $k\left(E^{0}\right)$, to be expected because of the greater density of states in this isotopically substituted molecule.

The results of the experiments demonstrate clearly the quantization in the transition state of dissociating ketene. A logical follow up will be to compare the experimental results with detailed calculations of $k(E)$, based on accurate computations of reagent state densities and transition state quantum levels. In this way, and perhaps with still higher resolution in the experiments, it may be possible to uncover non-RRKM behaviour ${ }^{3}$ associated, for example, with some breakdown in the entirely statistical base of RRKM theory.

There is little doubt that Moore's work will stimulate others to similar efforts. Indeed, already a paper ${ }^{4}$ from Wittig's laboratory at the University of Southern California reports observations of the steplike variation of $k(E)$ in the photoinitiated decomposition of $\mathrm{NO}_{2}$. The small size of this reagent, and consequent reduction in $\rho(E)$, means that its unimolecular lifetime just above $E^{0}$ is in the picosecond range, requiring the use of subpicosecond laser pulses for excitation and observation (in this case of NO). The size also brings an important benefit, as it simplifies the quantum level structure in the transition state, although the interpretation is clouded by extensive mixing of electronic states at energies sufficient for dissociation.

Other reactions which suggest themselves as targets for this kind of study are those with tightly constrained transition states, so that the quantum levels in the transition state, at least close to threshold, are widely spaced. Fourcentre unimolecular decompositions, such as the elimination of hydrogen halides from halogenated alkanes and alkenes, are prototypical of such reactions. They occur via transition states which should have easily resolvable structure $^{5}$. Whether the other conditions, which have been so successfully satisfied in the experiments in Moore's and Wittig's laboratories, can be satisfied in these other molecular systems remains to be seen.

Ian W. M. Smith is in the School of Chemistry, University of Birmingham, PO Box 363, Birmingham B15 2TT, UK.

1. Dantus, M., Rosker, J. J. \& Zewail, A. H. J. chem. Phys. 87, 2395-2397 (1987)

2. Lovejoy, E. R., Kim, S. K. \& Moore, C. B. Science 256 1541-1544 (1992).

3. Marcus, R. A. Science 256, 1523-1524 (1992)

4. Brucker, G. A., Ionov, S. I., Chen, Y. \& Wittig, C. Chem Phys. Lett. 194, 301-308 (1992)

5. Kato, S. \& Morokuma, K. J. chem. Phys. 73, 3900-3914 (1980); 74, 6285-6296 (1981).

6. Allen. W. D. \& Schaefer III, H. F. J. chem. Phys. 89. 329-341 (1988) 